\title{
Test Results of the Plasma Source for Underdense Plasma Lens Experiments at the UCLA Neptune Lab
}

\author{
H. Suk, C. E. Clayton ${ }^{\dagger}$, R. Narang ${ }^{\dagger}$, P. Muggli ${ }^{\dagger}$, \\ J. Rosenzweig, C. Pellegrini, and C. Joshi ${ }^{\dagger}$ \\ Department of Physics and Astronomy \\ University of California, Los Angeles, CA 90095 \\ $\dagger$ Department of Electrical Engineering
}

\begin{abstract}
A plasma source was developed at UCLA for planned underdense plasma lens experiments, where the plasma density is less than the electron beam density. The argon plasma, produced by a discharge between a $\mathrm{LaB}_{6}$ cathode at $1330{ }^{\circ} \mathrm{C}$ and a tantalum anode, is confined by a solenoidal magnetic field and flows transversely across the electron beam path. Extensive test of the plasma source is under way for various conditions before it is assembled with the UCLA photocathode-based electron linac. In particular, different longitudinal (with respect to the electron beam) plasma profiles and effective plasma lengths can be obtained by adjusting the moveable sliding door between the plasma source and the transverse beamline. Test results of the plasma source are presented.
\end{abstract}

\section{Introduction}

Plasma lenses have potential applications to next generation linear colliders because plasma focusing can be several orders of magnitude stronger than that of conventional magnets $[1,2]$. For this purpose, numerous studies have been done both in theory and experiment $[3,4,5,6,7]$. In the experimental area, the plasma lens research has been focused on the overdense plasma regime where the plasma density is larger than the beam density. In the case of overdense plasma lenses, however, a plasma return current flows inside the beam region when the beam propagates in the plasma. Hence, overdense plasma lenses lead to rather large spatial and temporal aberrations. In contrast, in underdense plasma lenses most of the plasma electrons are radially expelled from the beam 


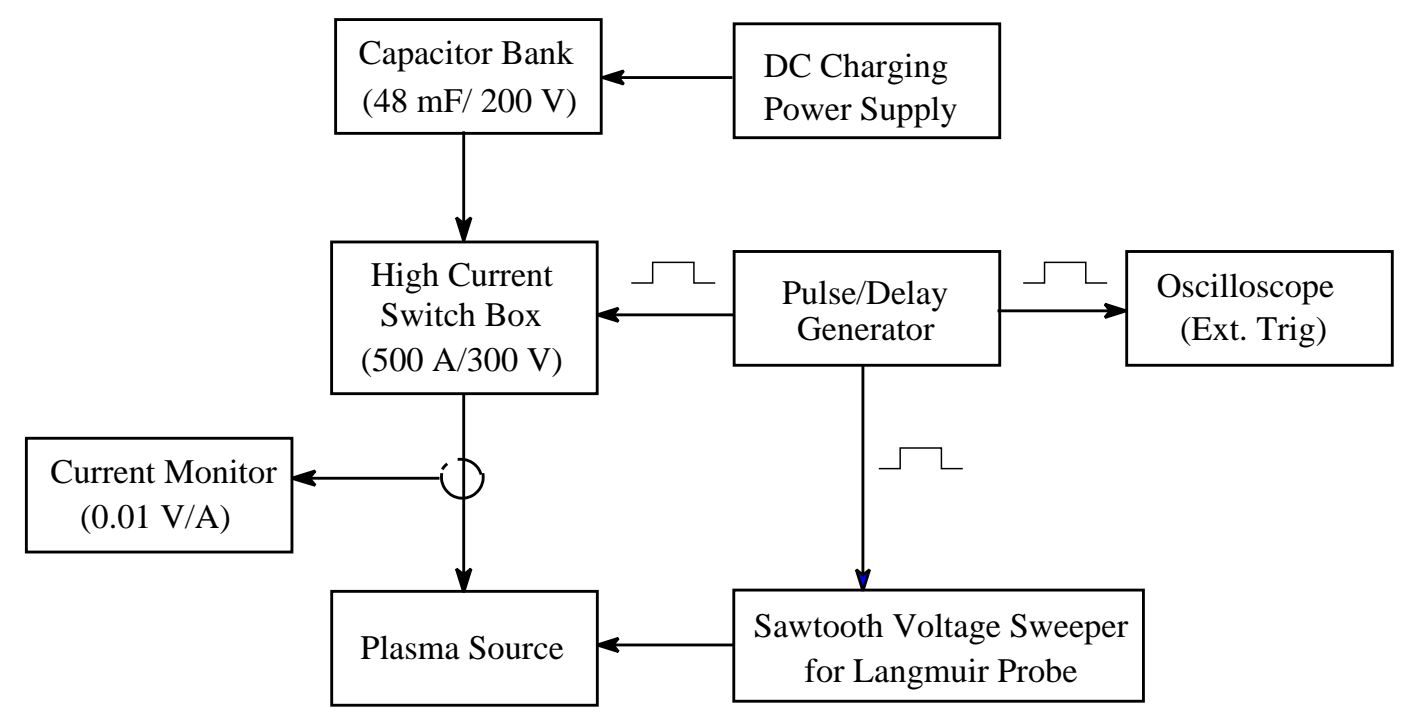

Figure 1: Block diagram of the plasma source test.

path when the beam propagates in the plasmas. Therefore, a nearly uniform electron-free ion channel is produced and this ion channel provides a high quality focusing. In other words, the focusing force is linear in radius and independent of the longitudinal position so that aberrations are avoided in underdense plasma lenses. This focusing effect was observed in UCLA/ANL experiments where the plasma was thick, compared to the focal length [8]. For practical underdense plasma lenses, however, the plasma thickness should be less than the focal length and this kind of thin underdense plasma lenses have not been explored experimentally so far. Hence, experiments in this regime are planned at the UCLA Neptune laboratory [9] which is based on the 1.625-cell photoinjector RF gun and the PWT linac. For this underdense plasma lens experiment, a discharge plasma source has been developed and tested at UCLA. In this paper, details of the plasma source and test results are presented.

\section{Description of the Plasma Source}

Figure 1 shows a block diagram of the setup for testing the plasma source. The capacitor bank consists of four $12 \mathrm{mF}$ capacitors connected in parallel. 


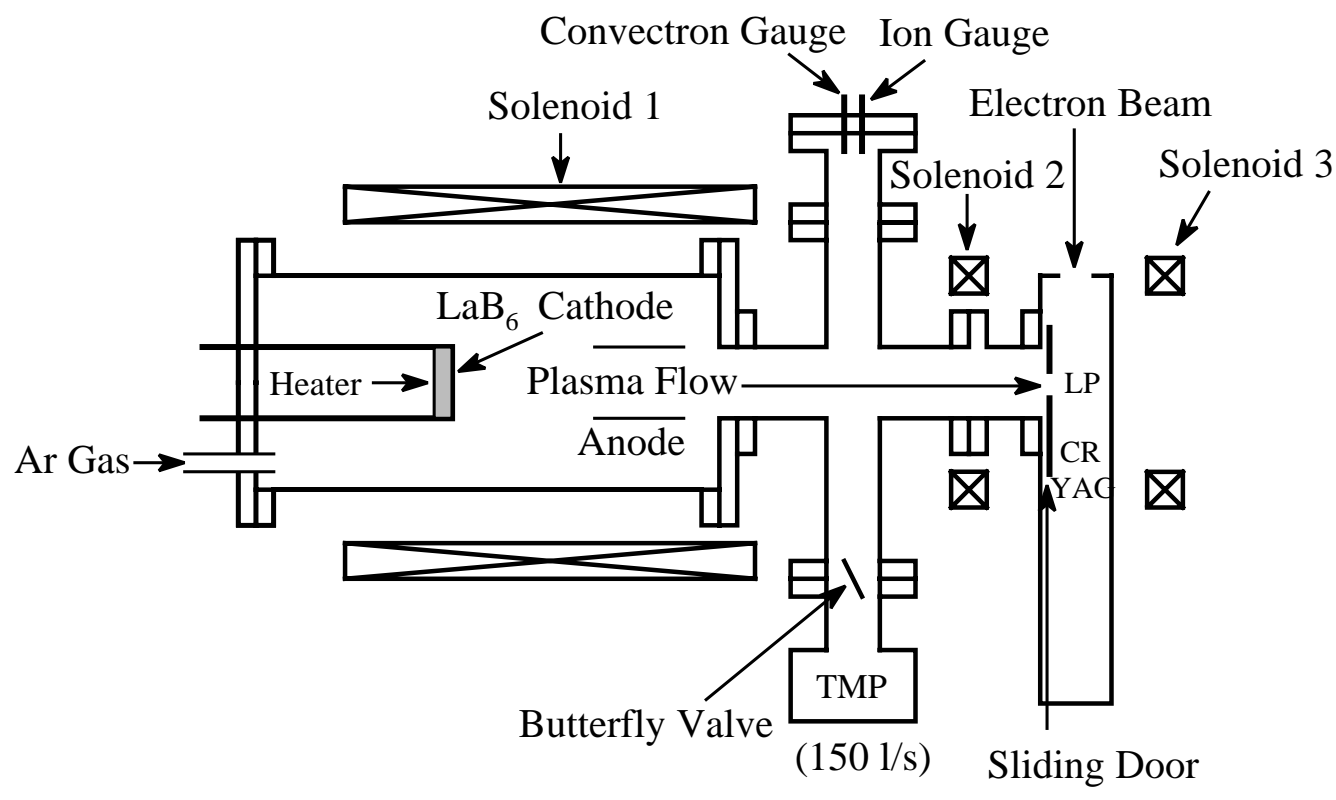

LP : Langmuir probe

$\mathrm{CR}$ : Cherenkov radiator

YAG : YAG crystal

Figure 2: Schematic drawing of the plasma source.

It is charged by the DC charging power supply through a $33.5 \Omega$ resistor and the charging $\mathrm{RC}$ time constant for this capacitor bank is $1.6 \mathrm{sec}$. For plasma generation, the high-current switch box (up to $500 \mathrm{~A} / 300 \mathrm{~V}$ ) is switched on by a trigger signal from the pulse/delay generator and the charged capacitor bank is discharged. This discharge current is measured with a current monitor with a sensitivity of $0.01 \mathrm{~V} / \mathrm{A}$. At the same time, a voltage sweeper provides a sawtooth voltage to the Langmuir probe for plasma diagnostics. The sawtooth voltage signal is adjustable between $-120 \mathrm{~V}$ and $120 \mathrm{~V}$. Figure 2 shows a detailed schematic drawing of the plasma source. As shown in the figure, the $\mathrm{LaB}_{6}$ cathode with a diameter of $7 \mathrm{~cm}$ is indirectly heated by a tungsten wire heater up to $1330{ }^{\circ} \mathrm{C}$ and the discharge pulse from the capacitor bank is applied between the tantalum hollow anode and the grounded cathode, where the separation is $6.7 \mathrm{~cm}$. As a result, an electric discharge occurs and a plasma is generated because the plasma source is filled with an argon gas in mTorr range.

Figure 3 shows a typical discharge current pulse (bottom trace) and a saw- 


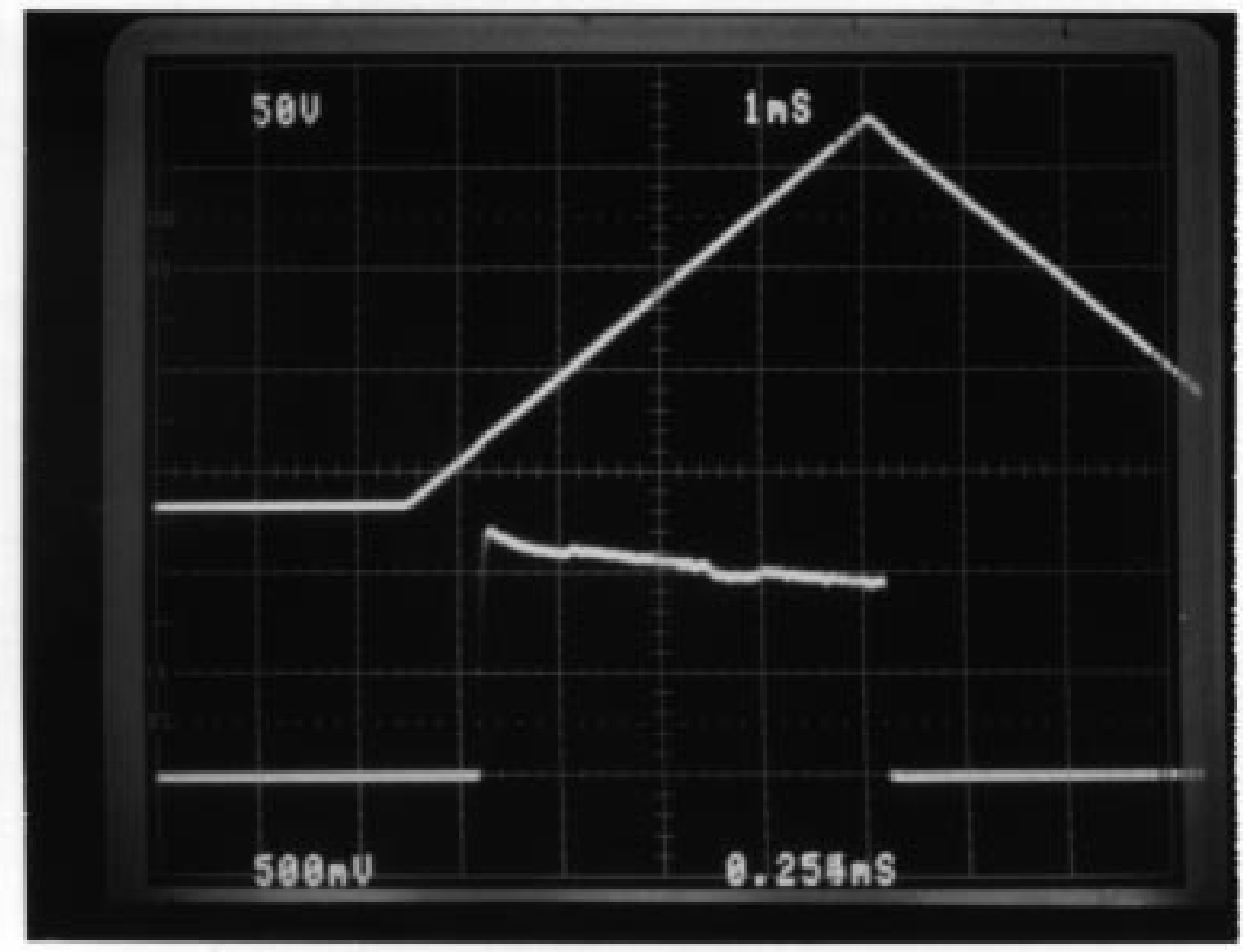

Figure 3: Typical discharge current pulse (bottom trace) and sawtooth voltage signal from the voltage sweeper (top trace). The discharge current pulse was obtained for $\mathrm{P}=2 \mathrm{~m}$ Torr, $\mathrm{V}_{d}=90 \mathrm{~V}$ and $\mathrm{T}_{c}=1280{ }^{\circ} \mathrm{C}$.

tooth voltage (top trace) applied to the Langmuir probe. The current is about $100 \mathrm{~A}$ and its duration is $4 \mathrm{~ms}$. For the current measurement, a discharge voltage of $90 \mathrm{~V}$ and a pressure of 2 mTorr were used when the cathode temperature was $1280^{\circ} \mathrm{C}$. Figure 3 also shows that the sawtooth voltage applied to the Langmuir probe is synchronized with the discharge current and it sweeps from $-120 \mathrm{~V}$ to $75 \mathrm{~V}$ in $4.5 \mathrm{~ms}$. The generated plasma is guided by a solenoidal magnetic field to flow towards the long diagnostic chamber. As shown in Fig. 2 , a sliding door is located at the entrance of the long diagnostic chamber and the plasma thickness traversed by the electron beam can be controlled by adjusting the sliding door gap. The gap can be changed from 0 to $7.5 \mathrm{~cm}$. Hence, different longitudinal plasma profiles can be obtained in the long diagnostic chamber, as will be shown below. In the beam-plasma interaction region, a Langmuir probe, which consists of a ceramic tube and a tungsten wire with a $1.43 \mathrm{~mm}^{2}$ surface area, is installed to measure the plasma density and the 
electron temperature. The Langmuir probe is moveable in the direction of the high energy electron beam path so that it can be used to measure longitudinal plasma density profiles for various conditions. When a high energy electron beam passes through the plasma lens, it is focused downstream and the focal point is located outside the plasma in the case of a thin lens. To get information about time-resolved beam dynamics a Cherenkov radiator is installed in the long diagnostic chamber. In addition, a YAG crystal is located in the chamber to measure the beam size.

\section{Test Results}

The plasma source has been tested for various conditions. As expected, the plasma density and its longitudinal profile in the diagnostic chamber are determined by many experimental parameters, such as gas pressure, discharge current/voltage, cathode temperature, magnetic fields of the solenoids, and sliding door gap. These variables were changed consecutively to find an optimum condition for plasma lens experiments.

First, the magnetic fields of the three solenoids were changed up to $70 \mathrm{G}$ to study the effect of magnetic fields. It was found that the main solenoid in cathode-anode region, denoted by Solenoid 1 in Fig. 2, does not make a significant difference in discharge current and plasma density, while the magnetic field by the two small solenoids, denoted by Solenoid 2 and Solenoid 3 in Fig. 2, significantly affects the plasma density. However, the magnetic field strength in the long diagnostic chamber can not be increased too much because it bends the electron beam path transversely when the beam propagates in the magnetic field region. Hence, there is a certain limit in magnetic field strength. For this reason, the magnetic field in the plasma lens region was set between 50 to $70 \mathrm{G}$. In this magnetic field region, the plasma density turned out to be large enough for plasma lens experiments. For a fixed magnetic field at the location of the plasma lens, the magnetic field of Solenoid 1 was adjusted to obtain a maximum plasma density in the plasma lens region. It was observed that a maximum plasma density is achieved when the magnetic field is almost uniform from the cathode-anode region to the plasma lens region.

To investigate the dependence of the discharge current on the discharge voltage, pressure and cathode temperature, these parameters were varied. Figure 4 (a) shows the discharge current vs. discharge voltage in the pressure range of 0.5 to $8 \mathrm{~m}$ Torr when the cathode temperature $T_{c}$ and the magnetic field are $1280{ }^{\circ} \mathrm{C}$ and $53 \mathrm{G}$, respectively. The discharge current increases as the voltage 

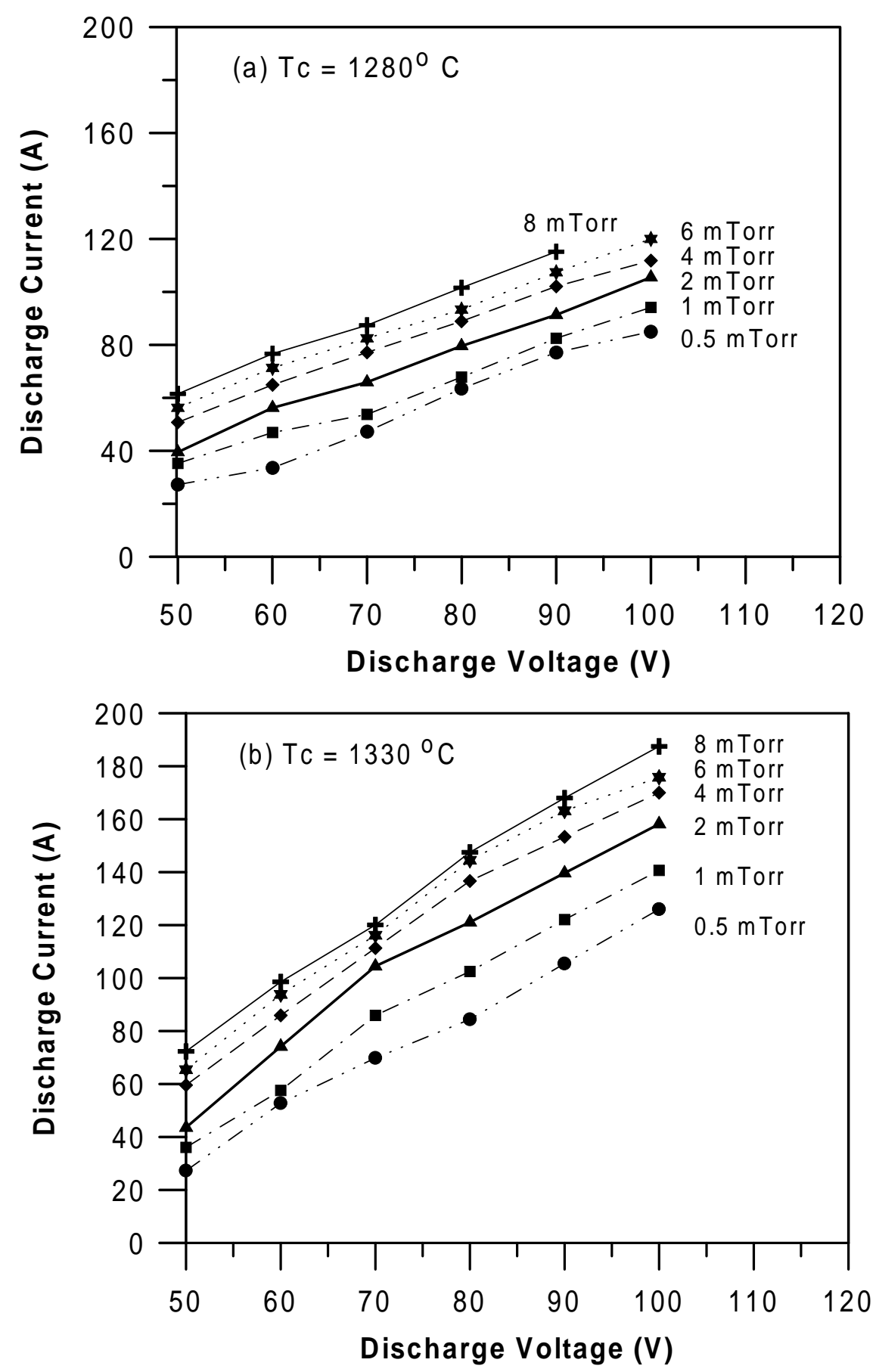

Figure 4: Discharge current vs. discharge voltage for different pressures and different cathode temperatures. (a) $\mathrm{T}_{c}=1280{ }^{\circ} \mathrm{C}$, (b) $\mathrm{T}_{c}=1330{ }^{\circ} \mathrm{C}$. 
increases for each pressure, and the discharge current also increases when the pressure is increased. However, it should be noted that the effect of pressure on the discharge current is small, compared to that of the discharge voltage. This is because the discharge current is dominated by seed thermal electrons emitted from the cathode. When the discharge voltage was increased beyond $110 \mathrm{~V}$, an arc began to occur and it was more easily produced in the higher pressure range. During arcing, the discharge current increased a lot, but the plasma density in the long diagnostic chamber was observed to remain very low. In order to investigate the effect of the cathode temperature, it was raised to $1330{ }^{\circ} \mathrm{C}$. The result for this case is shown in Fig. 4(b). It shows that the discharge current increases significantly, compared to Fig. 4(a). For 2 mTorr and $100 \mathrm{~V}$, for example, the discharge current increases from $105 \mathrm{~A}$ to $158 \mathrm{~A}$ as the cathode temperature is raised (i.e., a $50 \%$ current increase was observed). Furthermore, arcing was suppressed noticeably when the cathode temperature was higher.

Plasma densities were measured along diagnostic chamber for different discharge voltages and pressures. Figure 5(a) shows the density measurement when the cathode temperature and the sliding door gap were $1330{ }^{\circ} \mathrm{C}$ and $2.5 \mathrm{~cm}$, respectively. The figure shows that the plasma density increases for higher discharge voltages, as expected. It also shows that the three density curves have a maximum around 2 mTorr. This is due to a larger diffusion rate for a higher pressure, i.e., a diffusion rate is proportional to the pressure during transport from the cathode-anode region to the diagnostic chamber, while a plasma production is not proportional to the pressure for a given discharge voltage, as shown in Fig. 4(b). Therefore, the density will decrease beyond a certain pressure. Electron temperature was also measured for different discharge voltages and pressures. Figure 5(b) shows a graph of the electron temperature vs. pressure in the case of $V_{d}=100 \mathrm{~V}$. The figure shows that the electron temperature decreases from $7.1 \mathrm{eV}$ to $3.5 \mathrm{eV}$ as the pressure increases.

Plasma density profiles along the beam direction were measured for different conditions to find an optimum condition for the plasma lens experiment. Two cases are shown in Fig. 6(a) and Fig. 6(b). In Fig. 6(a), the sliding door gap is $1.5 \mathrm{~cm}$ and the discharge voltage is $100 \mathrm{~V}$. In this case, the peak plasma density and the plasma thickness are too large for underdense plasma lens experiments, as shown in the next section. Hence, the gap was changed to $1 \mathrm{~cm}$ to reduce the peak plasma density and the plasma thickness. In this case, however, the peak density was observed to decrease too much so that the discharge voltage was increased to $110 \mathrm{~V}$ to compensate the density decrease. 

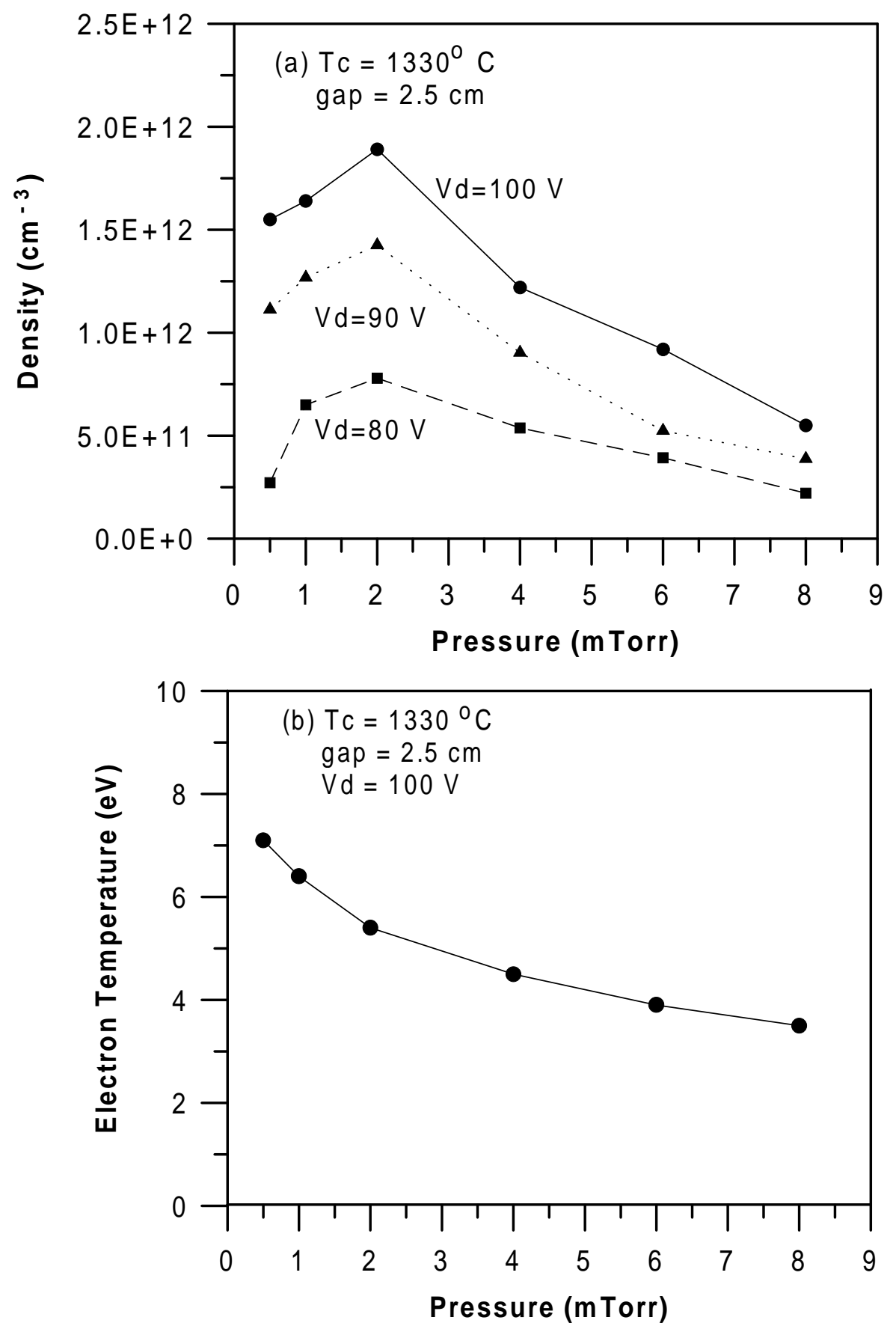

Figure 5: (a)Plasma density vs. pressure for different discharge voltages. The plasma density is measured at the long diagnostic chamber. (b) Electron temperature vs. pressure for $\mathrm{V}_{d}=100 \mathrm{~V}$. 

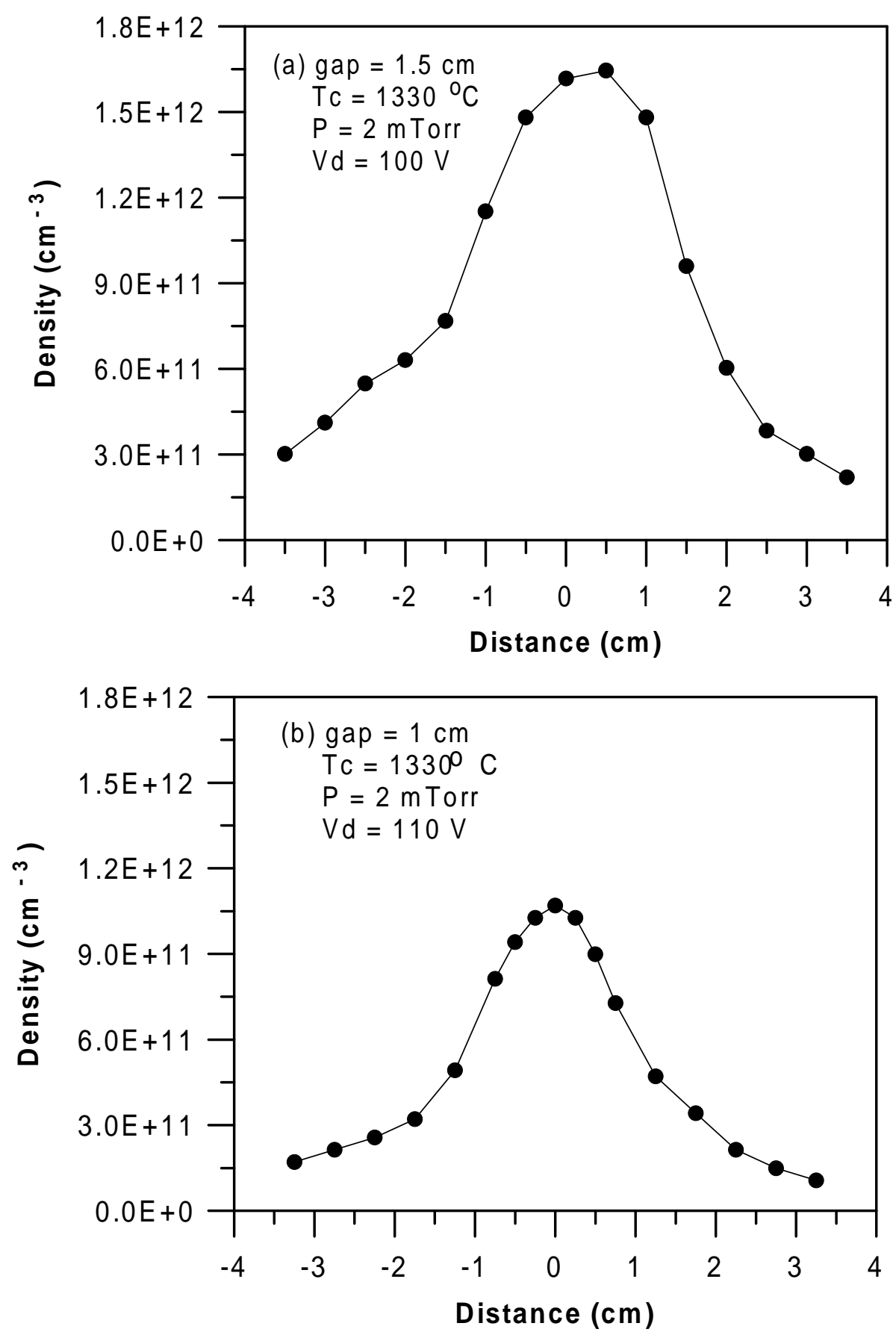

Figure 6: Plasma density profiles along the beam direction. (a) gap $=1.5 \mathrm{~cm}$, $\mathrm{V}_{d}=100 \mathrm{~V}$, (b) gap $=1 \mathrm{~cm}, \mathrm{~V}_{d}=110 \mathrm{~V}$. 
Fig. 6(b) shows this case. The graph shows that the peak plasma density $n_{p}$ is about $1.1 \times 10^{12} \mathrm{~cm}^{-3}$ and the effective length of the plasma lens, which is defined by $L_{p}=\int f(z) d z / n_{p}$, is about $2.9 \mathrm{~cm}$. Here, $f(z)$ is the density profile function. For these plasma parameters all requirements for underdense plasma lens experiments are satisfied, as shown in the next section.

\section{Experimental Parameters}

Experiments on the underdense plasma lens will be conducted at the UCLA Neptune laboratory. The basic parameters for the planned plasma lens experiment are summarized in Table 1, where the plasma parameters are based on the data shown in Fig. 6(b).

Table 1 : Experimental parameters.

$=========================$

Electron beam energy $E=16 \mathrm{MeV}$

Charge per bunch $Q=5 \mathrm{nC}$

Normalized beam emittance $\epsilon_{n}=20 \mathrm{~mm}-\mathrm{mrad}$

Beam duration $\sigma_{t}=25 \mathrm{ps}$

Beam radius (rms) at plasma entrance $\sigma_{i}=400 \mu \mathrm{m}$

Beam density $n_{b}=3.2 \times 10^{12} \mathrm{~cm}^{-3}$

Peak plasma density $n_{p}=1.1 \times 10^{12} \mathrm{~cm}^{-3}$

Effective plasma lens length $L_{p}=2.9 \mathrm{~cm}$

As shown in the table, the beam density $n_{b}$ is larger than the peak density of the plasma lens, i.e., the plasma is underdense. Furthermore, the quantity $k_{p} \sigma_{z} \sqrt{n_{b} / n_{p}}$ [9], where $k_{p}$ is given by $k_{p}=\sqrt{4 \pi r_{e} n_{p}}$, is calculated to be 2.5 so that the electron bunch length $\sigma_{z}$ is long enough to expel plasma electrons from the beam path. Here, the parameter $r_{e}$ is the classical radius of an electron. The focal length $f$ of this plasma lens can be calculated by $f=2 \gamma c^{2} / L_{p} \omega_{p}^{2}$ if the thin lens approximation, i.e., $L_{p}<f$, is satisfied [10]. In this equation, $\gamma$ is the Lorentz factor and $c$ is the velocity of light. For the parameters of Table 1, the focal length $f$ is calculated as $5.7 \mathrm{~cm}$ and is larger than the effective plasma length. Hence, the thin lens approximation is satisfied. For a plasma lens to be useful, its length should be less than $\lambda_{\beta} / 4$, where $\lambda_{\beta}$ is the betatron wavelength given by $\left(2 \pi \gamma / r_{e} n_{p}\right)^{1 / 2}$. Otherwise, the beam emittance 
will increase significantly. In the case of Table $1, \lambda_{\beta} / 4$ is calculated as $6.4 \mathrm{~cm}$ and this is larger than $L_{p}$. Hence, the requirement of $L_{p}<\lambda_{\beta} / 4$ is satisfied.

The beam radius at the focal point, $\sigma_{f}$, can be estimated for the parameters given in this section. The $\beta$-function at the entrance of the plasma lens, $\beta_{0}$, is calculated as $25.1 \mathrm{~cm}$, and $\beta_{0}^{2}$ turns out to be much larger than $f^{2}$. In this case, the beam radius at the focal point is approximately given by $\sigma_{f} \simeq f \epsilon_{n} / \gamma \sigma_{i}$ [10], where $\sigma_{i}$ is the initial beam radius at the entrance of the plasma lens, and $\sigma_{f}$ is calculated to be $91 \mu \mathrm{m}$. Therefore, the beam size will be reduced by a factor of 4.4, as a result of plasma focusing. The beam size and the timeresolved beam profile will be measured at different positions along the beam path by using the YAG crystal, the Cherenkov radiator, and a psec-resolution streak camera.

In this plasma lens experiment, there is one potential problem related with bending of the electron beam path. When the beam propagates in the plasma lens region, its path will be bent because of the transverse magnetic field from the solenoids. To investigate this issue, the transverse magnetic field was measured along the beam path and this result was used to calculate the beam trajectory. In Fig. 7, the measured magnetic field at different positions is represented by the round dots and a Gaussian profile is used for best fitting. The resulting Gaussian function was used for calculation of the beam trajectory. According to this calculation, the beam is deflected in the magnetic field region, as represented by the dotted line in the figure. The calculation shows that the beam is deflected by $1.3 \mathrm{~mm}$ at the position of the focal point $(f=5.7$ $\mathrm{cm}$ ) and deflected by $4 \mathrm{~mm}$ at a position of $20 \mathrm{~cm}$ downstream the plasma lens. This will be a problem for Cherenkov radiation measurements. Hence, a couple of methods, such as adding a special iron structure to reconfigure the magnetic field shape, using a small Helmholtz coil to compensate for the magnetic field, etc., will be investigated to solve this problem.

\section{Summary}

A $\mathrm{LaB}_{6}$-based discharge plasma source was developed for thin underdense plasma lens experiments at UCLA and it was tested extensively for various experimental conditions. The results show that the plasma source can provide satisfactory plasma parameters for thin underdense plasma-lens experiments, i.e., plasma densities in low $10^{12} \mathrm{~cm}^{-3}$ range and an effective length of a few $\mathrm{cm}$. The plasma source is expected to be assembled with the Neptune Laboratory beamline by the end of this year, after beam characterizations of the new 


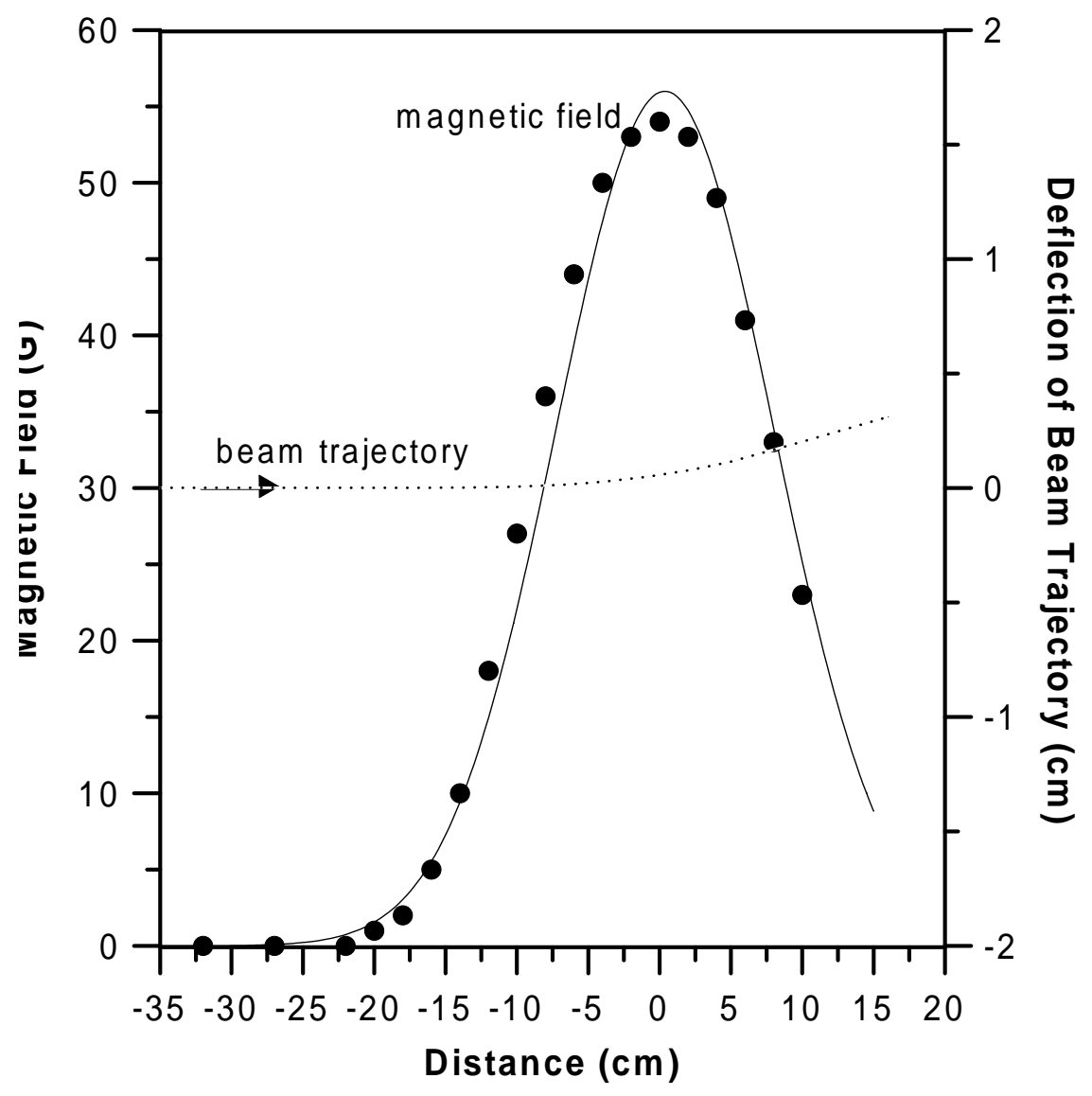

Figure 7: Measured magnetic field profile along the beam direction (round dots) and calculated deflection of the beam trajectory (dotted line) for $\mathrm{E}=16$ $\mathrm{MeV}$. The solid line is a Gaussian fit to the measured magnetic field.

1.625-cell photoinjector gun.

\section{Acknowledgements}

The authors of the paper would like to thank Dr. G. Hairapetian for construction and initial measurements of the prototype for the plasma source. 


\section{References}

[1] P. Chen, Part. Accel. 20, 171 (1987).

[2] T. Katsouleas et al., Phys. Fluids B 2, 1384 (1990).

[3] W. E. Martin et al., Phys. Rev. Lett. 54685 (1985).

[4] N. Barov and J. Rosenzweig, Phys. Rev. E 49, 4407 (1994).

[5] H. Nakanishi et al., Phys. Rev. Lett. 66, 1870 (1991).

[6] J. J. Su et al., Phys. Rev. A 41, 3321 (1990).

[7] G. Hairapetian et al., Phys. Rev. Lett. 72, 2403 (1994).

[8] N. Barov et al., Phys. Rev. Lett. 80, 81 (1998).

[9] J. Rosenzweig et al., Nucl. Intrs. Meth. A 410, 437 (1998).

[10] J. Davis, Ph.D. Dissertation, Dept. of Electrical Engineering, UCLA (1996). 